\title{
Skin Sympathetic Nerve Activity and Rate Control of Atrial Fibrillation
}

\author{
Anthony Douglas II, Takashi Kusayama, MD, PhD, Peng Sheng Chen M.D. \\ The Krannert Institute of Cardiology and Division of Cardiology, Department of \\ Medicine, Indiana University School of Medicine
}

\section{Background and Hypothesis}

Lifetime risks for atrial fibrillation (AF) are 1 in 4 for people 40 years of age and older. Rate and rhythm control are both important strategies in managing patients with AF. However, not all patients respond to beta blocker therapy. We hypothesize that sympathetic tone is important in rate control of AF, but the importance varied among patients.

\section{Experimental Design or Project Methods}

neuECG is a new noninvasive method to record skin sympathetic nerve activity (SKNA) and electrocardiogram. The present research recruited and recorded 2 paroxysmal and 3 persistent AF patients for 24 hours using neuECG. The average voltage of SKNA (aSKNA) and ventricular rate during AF was analyzed in one-min windows.

\section{Results}

The preliminary data reveal strong correlations $(r=.783, r=.640$, respectively, $p<0.0001$ for both) between aSKNA $(1.19 \pm 0.21 \mu \mathrm{V})$ and ventricular rate $(110 \pm$ $8 \mathrm{bpm}$ ) in the paroxysmal AF. High correlations were consistently observed when data were analyzed on an hourly basis. In comparison, strong correlations were found between aSKNA $(1.13 \pm 0.2 \mu \mathrm{V})$ and ventricular rate $(88 \pm 8.6 \mathrm{bpm})$ in persistent $A F(r=.496, r=.796, r=.636$ respectively, $p<0.001$ for both cases). However, hourly correlations displayed much higher variability between aSKNA and ventricular rate than that observed for paroxysmal AF.

\section{Conclusion and Potential Impact.}

aSKNA positively correlates with ventricular rate during AF. This relationship is stronger and more consistent during paroxysmal than persistent AF. These findings may be important in determining the efficacy of beta blocker therapy in rate control of $A F$. 